Z Rheumatol $2017 \cdot 76: 292-294$

DOI 10.1007/s00393-017-0284-6

Online publiziert: 3. März 2017

๑) Springer-Verlag Berlin Heidelberg 2017

CrossMark

\section{G. Horneff' $\cdot$ R. E. Schmidt ${ }^{2}$}

'Asklepios Kinderklinik Sankt Augustin, Sankt Augustin, Deutschland

${ }^{2}$ Klinik für Immunologie und Rheumatologie, Medizinische Hochschule Hannover, Hannover, Deutschland

\title{
Genetik bei autoinflammatorischen Syndromen
}

Autoinflammatorische Syndrome sind seltene, i.d.R. monogen erbliche Erkrankungen, gekennzeichnet durch multisystemische Entzündungsprozesse oft mit rekurrierenden Fieberattacken, aber auch mit anderen, klinisch wenig spezifischen Manifestationen. Betroffen sein können die Haut, das muskuloskeletale System, Lymphknoten, Abdomen und auch das zentrale Nervensystem und die Sinnesorgane. Charakteristische Autoimmunphänomene wie Autoantikörper oder autoantigenspezifische T-Zellen werden in der Regel nicht nachgewiesen oder sind sogar irreführend.

\section{) Genetische Diagnostik hilft bei der Abgrenzung von nicht ge- netisch bedingten Erkrankungen mit Autoinflammation}

Weil die Anzahl genetisch identifizierter Erkrankungen wie auch die methodischen Optionen stetig zunehmen, erhält die genetische Diagnostik eine wachsende Bedeutung zur Abgrenzung von nicht genetisch bedingten Erkrankungen mit Autoinflammation, wie $z$. B. die systemische juvenile idiopathische Arthritis (Still-Syndrom), das PFAPA(periodisches Fieber, aphthöse Stomatitis, Pharyngitis, zervikale Adenitis)- sowie das SAPHO(Synovitis,Akne, Pustulosis, Hyperostose und Osteitis)Syndrom und die CRMO (chronisch rekurrierende multifokale Osteomyelitis). Diese Erkrankungen sind sämtlich dadurch charakterisiert, dass es zu einer gestörten Aktivierung von Inflammaso- men und damit vermehrten Expression von Interleukin-1 und anderen proinflammatorischen Zytokinen kommt. Mit den Interferonopathien wurde unlängst eine völlig neue Gruppe von autoinflammatorischen Erkrankungen mit einem gänzlich neuen immunologischen Hintergrund hinzugefügt. Kollege Fiehn stellt mittels einer erweiterten Kasuistik verschiedene genetisch bedingte Regulationsstörungen im Typ-1Interferon-Signalweg zusammen. Zu den Interferonopathien zählen auch die Proteasomopathien, die von Feist, Brehm, Kallinich und Krüger in ihrem Beitrag näher beleuchtet werden.

Klinisch lassen sich zahlreiche autoinflammatorische Erkrankungen in der Regel zunächst gut voneinander abgrenzen. Hilfreich sind das Alter beim Auftreten, die Fieberdauer und die Begleit- und Leitsymptome und oft auch der familiäre Hintergrund. Kurzes, wenige Tage dauerndes Fieber begleitet von Bauchschmerzen seit dem Vorschulalter bei einem türkischen Kind legen ein familiäres Mittelmeerfieber (FMF) nahe. Und ein Kind mit tagelangen Fieberschüben seit frühester Kindheit, begleitet von Urtikaria und zunehmender Innenohrschwerhörigkeit, hat mutmaßlich ein Muckle-Wells-Syndrom. Was aber, wenn das Kind mit mutmaßlichem FMF nur eine pathogene Mutation im Pyringen aufweist? Wann ist schon bei Vorliegen typischer Klinik für ein FMF, aber genetischer Heterozygotie mit nur einer pathogenen FMF-Mutation die Diagnose FMF richtig, die weitere genetische Diagnostik unnötig und eine Therapie zu starten? Wann ist sie „lebenslang“ notwendig und wann nicht? Aufklärung zu diesem Thema enthält der Betrag von Kallinich.

Was aber, wenn bei dem oben genannten Kind mit mutmaßlichem FMF und nur einer pathogenen Mutation im Pyringen die Fieberschübe gegenüber Colchicin aber refraktär sind und das Kind auch eine CAPS-Genmutation aufweist, wie im Falle des Kindes ein Basenaustausch mit unklarer Bedeutung?

Die Detektion solcher Konstellationen ist nur unter Einsatz von genetischer Diagnostik möglich. Das Angebot seitens der Humangenetiker ist groß und kann nach einem Algorithmus genutzt werden, das klinisch wahrscheinlichste Gen zuerst, und, wenn man nicht fündig wurde, wird in einem Gen nach dem anderen sequenziert. Oder soll gleich eine Paneldiagnostik erfolgen, die gleichzeitige Untersuchung von z. B. 20 „häufigeren“, aber allesamt ziemlich selten auftretenden Gendefekten, die mit der Aussage beworben wird, in bis zu $80 \%$ der klinisch diagnostizierten, wahrscheinlich genetisch verursachten Erkrankungen wird keine ursächliche Genveränderung gefunden? Die Möglichkeiten der Gendiagnostik werden weiter und weiter, nahezu grenzenlos. Frau Lee-Kirsch gibt einen Überblick über die aktuell verfügbare genetische Methodik, die sich von der Sanger-Sequenzierung sehr weiterentwickelt hat.

Doch hat die genetische Diagnostik auch ihre Fallgruben. So sind keineswegs alle genetischen Defekte klinisch gut charakterisierter Erkrankungen bekannt. Bei Auftreten monogenetischer Erkrankungen innerhalb einer Familie mit der glei- 
Hier steht eine Anzeige.

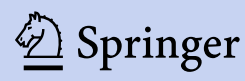


chen Mutation kann die klinische Manifestation sehr unterschiedlich sein, ein Phänomen, das als verminderte Penetranz bekannt ist. Dies wirft die Frage der Genotyp-Phänotyp-Korrelation auf, oftmals ist sie schwach.

Manche genetischen Varianten des autosomal-dominant vererbten Tumornekrosefaktorrezeptor-assoziierten periodischen Syndroms sind mit einem milderen Verlauf oder einer verminderten Penetranz assoziiert, bei manchen dagegen bestimmt die genaue Art der Mutation das Therapieansprechen oder auch die medikamentös induzierte Schubinduktion.

Anders herum können sog. SingleNucleotid-Polymorphismen (SNP) vorliegen, ohne für die Erkrankung verantwortlich zu sein. Das sind nichtpathogene Mutationen, die in höherer Häufigkeit auch bei Gesunden zu finden sind. Problematisch wird es, wenn solche, eigentlich als nicht pathogene SNP bekannte molekulare Defekte in verschiedenen Genen einen möglichen „Summationseffekt" bedingen. Wie ist das zu bewerten?

Bei autosomal-dominant vererbten Erkrankungen liegt bei klinisch und genetisch nicht betroffenen Eltern mit einem betroffenen Kind eine Neumutation vor. Wie häufig und wie bedeutsam sind aber somatische Mutationen? Bei somatischen Mutationen liegt ein Mosaik vor. Ein Teil der Zellen trägt das Genom der Keimbahn, ein Teil dagegen eine in einer Tochterzelle der befruchteten Eizelle entstandene Mutation. Ab welchem Verhältnis aber ist diese somatische Mutation relevant? Und kann diese auch in den Gameten bestehen und in die nächste Generation vererbt werden. Auf diese Problematik gehen die Autoren Kümmerle-Deschner und Lohse in ihrem Beitrag ein.

\section{》) Der klinisch tätige \\ Rheumatologe braucht Wissen um Möglichkeiten und Fallstricke genetischer Diagnostik}

Die Genetik hat längst begonnen, zumindest Teile der klinischen Rheumatologie und mehr noch der Kinderrheu- matologie zu beherrschen. Noch werden Diagnosen klinisch gestellt oder unter Einsatz von klinischen Diagnosekriterien. Trotzdem braucht der klinisch tätige Rheumatologe Wissen um Möglichkeiten und Fallstricke der genetischen Diagnostik.

In dieser Ausgabe der Zeitschrift für Rheumatologie vermitteln die Autoren solches Wissen. Wir wünschen Ihnen viel Spaß bei der Lektüre.

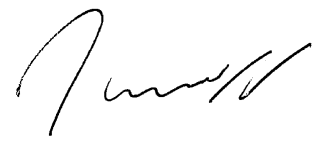

Prof. Dr. Gerd Horneff
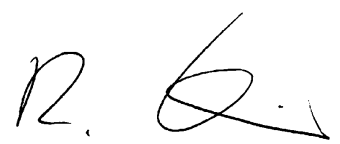

Prof. Dr. Reinhold E. Schmidt

\section{Korrespondenzadresse}

Prof. Dr. G. Horneff

Asklepios Kinderklinik Sankt Augustin

Arnold-Janssen-Str. 29, 53757 Sankt Augustin, Deutschland

g.horneff@asklepios.com

\section{Prof. Dr. R. E. Schmidt}

Klinik für Immunologie und Rheumatologie, Medizinische Hochschule Hannover Carl-Neuberg-Str. 1, 30625 Hannover, Deutschland

immunologie@mh-hannover.de

Interessenkonflikt. G. Horneff und R.E. Schmidt geben an, dass kein Interessenkonflikt besteht.
Unspezifischer Kreuzschmerz: NVL empfiehlt Akupunktur

DÄGfA begrüßt Aufwertung der Akupunktur

Aufgrund der zunehmenden wissenschaftlichen Evidenz wird die Akupunktur nun auch in der novellierten Nationalen VersorgungsLeitlinie (NVL) zur Therapie von Rückenschmerzen explizit empfohlen.

Die in die aktuelle Fassung der NVL aufgenommene Empfehlung, bei nicht-spezifischen Rückenschmerzen Akupunktur einzusetzen, begrüßt PD Dr. Dominik Irnich, 1. Vorsitzender der Deutschen Ärztegesellschaft für Akupunktur (DÄGfA): Damit finden nun auch die guten Studienergebnisse ihren Niederschlag in der Nationalen VersorgungsLeitlinie Nicht-spezifischer Kreuzschmerz. Irnich weist darauf hin, dass Akupunktur jedoch nur dann die optimale Wirkung entfaltet, wenn diese fachgerecht und gewissenhaft ausgeführt wird. Dies erfordert ein individuelles Eingehen auf den Patienten, eine ganzheitliche Sichtweise gerade beim chronischen Schmerz, eine qualitativ hochwertige Ausbildung und sichere Fertigkeiten, wie er betont.

Der Leiter des Wissenschaftszentrums (WissZ) der DÄGfA, Prof. Dr. Dr. Winfried Banzer, verwies in der Novellierungsphase der NVL darauf, dass die medikamentöse Therapie nicht grundsätzlich vor eine Akupunktur-Therapie gestellt werden sollte. Banzer sieht dafür keine wissenschaftliche Grundlage. Im Vergleich zu anderen empfohlenen Verfahren zeige die Akupunktur in aktuellen Studien bei einer Serie von 6 bis 12 Behandlungen eine bis zu einem Jahr anhaltende und starke Wirkung. Ärzte stehen nun in der Pflicht, ihre Patienten über die Wirkung der Akupunktur aufzuklären, so die DÄGfA. Zugleich sollten Patienten darüber informiert werden, dass die Qualität der Ausführung von besonderer Relevanz ist. Von der DÄGfA ausgebildete und geprüfte Ärzte sowie nähere Informationen zu spezifischen Qualifikationsnachweisen sind im Patientenportal unter www.daegfa.de aufgeführt.

Quelle: Deutsche Ärztegesellschaft für Akupunktur (DÄGfA) www.daegfa.de 